\title{
Upper-limb actuated exoskeleton for muscular dystrophy patients: preliminary results*
}

\author{
S. Dalla Gasperina, M. Gandolla, A. Manti, L. Aquilante, V. Longatelli, M.G. D’Angelo, F. Molteni, \\ E. Biffi, M. Rossini, M. Gfoehler, M. Puchinger, F. Braghin, A. Pedrocchi.
}

\begin{abstract}
Being able to perform a lost movement is an important experience towards increased independence and self-esteem, particularly for neuromuscular patients, who see their muscles weaken day after day. In this pilot study, preliminary results on the testing of a motorized upper-limb exoskeleton for muscular dystrophy patients are presented. The mechatronic system is a five Degrees of Freedom exoskeleton, which acts at shoulder, elbow, and wrist levels. It is designed to help severely impaired people to regain independence during daily-life activities. While wearing the exoskeleton, the user has the direct control of the system by actively piloting the position of endeffector by means of joystick or vocal control. The usability of the system and a quantitative assessment of arm functionality with and without the exoskeleton are evaluated on five muscular dystrophy patients. According to the objective functional benefit evaluation performed through the PUL scale, all participants strongly increased their range of motion and they were able to perform activities that were not possible without the exoskeleton, such as such as feeding, playing activities at the table, combing hair or using a keyboard. As for the evaluation of self-perceived functional benefit, four patients reflected the effective measured functional improvement. System usability has been evaluated to be good.
\end{abstract}

\section{INTRODUCTION}

Restore a lost function is a special experience for people affected by neuromuscular degenerative diseases, such as muscular dystrophy (MD), and, in this context, technology advancements can play an important role. Most effort has been devoted to the development and widespread of

\footnotetext{
Research supported by BRIDGE and EMPATIA projects funded by Fondazione Cariplo in collaboration with Regione Lombardia.

S. Dalla Gasperina, A. Manti, L. Aquilante, V. Longatelli, F. Braghin and A. Pedrocchi are with Politecnico di Milano, Milano, Italy. M. Gandolla is with Politecnico di Milano, Polo territoriale di Lecco, Lecco, Italy. M.G. D'Angelo and E. Biffi are with IRCCS E. Medea, Neuromuscular Unit, Department of Neurorehabilitation, Bosisio Parini, Italy. F. Molteni and M. Rossini are with Villa Beretta Rehabilitation Center, Valduce Hospital, Costamasnaga, Italy. M. Puchinger and $\mathrm{M}$. Gfoehler are with the Institute of Engineering Design and Logistics Engineering, TU Wien, Vienna, Austria.

M. Gandolla is the corresponding author (e-mail: marta.gandolla@polimi.it )
}

electrical wheelchairs and ventilators, so to assure autonomous mobility, and respiratory assistance, essential for survival [1]. However, with the overall increased life expectancy, upper extremity disability plays a key role in reducing patients' autonomy and worsening quality of life [2]. In 2007, the Dutch Duchenne Parent Project organized a workshop to determine whether improving arm or leg function should be prioritized according to people with Duchenne MD. Indeed, patients considered arm function as the highest priority. In fact, the loss of lower extremity function can be compensated fairly well by using a wheelchair, but compensating the loss of arm function is less evident [3]. MD is indeed characterized by multiple forms (e.g., Duchenne MD; Becker MD; Limb Girdle MD), but all are progressive and disabling over time given the gradual and continuous muscle wasting and weakening. Very little is known about severity, course and impact of upper limbs limitations in MDs, and only recently scales have been validated to detect modifications during time [4], but even less is known about adequate and effective aids able to reduce functional upper limb limitations. Commercially, only few motorized technologies are currently available. Devices such as Armon Ayura (Microgravity Products BV, The Netherlands), Neater Arm Support (Neater Solutions Ltd, United Kingdom), ExoArm or Darwing (Focal Meditech BV, The Netherlands) do provide arm support by means of a forearm brace, but since they still require user's muscular effort to fulfill the movements, they are not suitable for users with very poor functional residual ability [1], [5].

Pedrocchi and colleagues developed assistive [6] and rehabilitative [7] passive exoskeletons with the aim of exploiting any functional residual ability of severely impaired patients. However, given their reduced motor abilities, passive exoskeletons are not suitable for MD patients. The BRIDGE (Behavioural Reaching Interfaces during Daily antiGravity Activities through upper limb Exoskeleton) and EMPATIA@Lecco (EMpowerment del PAzienTe in cAsa) projects aim at developing a mechatronic upper limb exoskeleton for the assistance of daily life activities of MD people. The key philosophy of the projects is to contrast the everyday experience of MD people of losing functions by providing them with a system able i) to move their own arm to perform a desired action; and ii) to exploit their own residual capabilities to control their own arm in order to keep them partially autonomous as long and as much as possible. In this scenario, the BRIDGE/EMPATIA mechatronic system has been developed to contrast the severe upper-limb motion conditions of patients affected by 
Muscular Dystrophy that progressively lose their capabilities in arm movements. The system is composed by a lightweight arm exoskeleton, a set of human-machine interfaces for the control of the motion of the orthosis and a computer-based control unit aimed at computing the control algorithm by integrating the input from the user with the current position of the exoskeleton. When using the joystick, the user controls the exoskeleton hand position with the contralateral hand. When using the vocal control, it is not needed any residual motor abilities in the upper limbs. In this work, we present the preliminary results obtained by the testing of the system with the real end-users.

\section{MATERIALS AND METHODS}

\section{A. Mechatronic system}

BRIDGE/EMPATIA is a 5 Degrees of Freedom (DoFs) actuated exoskeleton for the upper limb which has been developed with the collaboration of TU Wien research group [8]. 3 DoFs are independently actuated at shoulder level (i.e. abduction and adduction, flexion and extension and internal and external rotation), while 1 DoF is actuated at the elbow level (i.e. flexion and extension) and $1 \mathrm{DoF}$ at the wrist (i.e. pronation and supination) (Figure 1). Each DoF is actuated by an electrical stepper motor, which is coupled to a Harmonic Drive (HD) reducer. The angular position of each joint is measured by an encoder, and it is limited both mechanically and electronically by means of physical stoppers and photocells, respectively. Every joint is characterized by a Range of Motion (RoM), which can be reduced according to the user's needs. The RoMs limit values are listed in Table I. The exoskeleton structure includes systems devoted to adapting the DOFs locations to the user's limb anthropometrics (i.e. arm and forearm lengths). The physical interfaces between the orthosis and the user are an ergonomic shell, which supports the arm, and a plate with

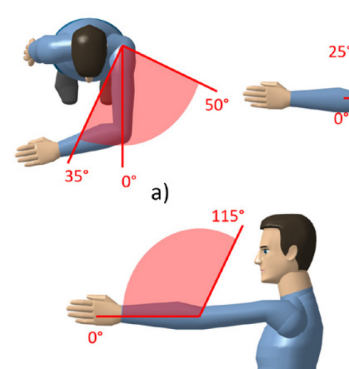

d)
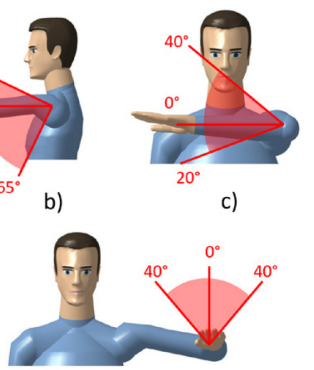

e)

Figure 1. Joints ROMs scheme. a) Shoulder abduction/adduction. b) Shoulder flexion/extension. c) Shoulder internal/external rotation. d) Elbow flexion/extension. e) Wrist pronation/supination

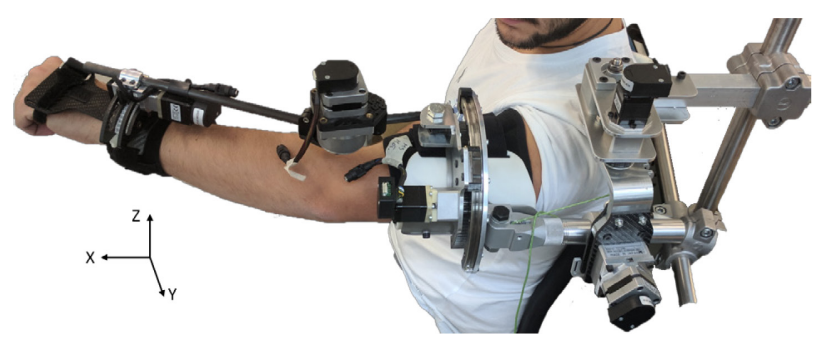

Figure 3. Mechatronic exoskeletal system
TABle I. JoInts Range Of Motion Limit VAlues.

\begin{tabular}{|c|c|c|c|}
\hline $\begin{array}{c}\text { Joint } \\
\text { number }\end{array}$ & Associated DOF & $\begin{array}{c}\text { Lower } \\
\text { limit [ }{ }^{\circ} \text { ] }\end{array}$ & $\begin{array}{c}\text { Upper } \\
\text { limit [ }{ }^{\circ} \text { ] }\end{array}$ \\
\hline 1 & $\begin{array}{c}\text { Shoulder } \\
\text { abduction/adduction }\end{array}$ & -50 & 35 \\
\hline 2 & Shoulder flexion/extension & -65 & 25 \\
\hline 3 & $\begin{array}{c}\text { Shoulder internal/external } \\
\text { rotation }\end{array}$ & -20 & 40 \\
\hline 4 & Elbow flexion/extension & 0 & 115 \\
\hline 5 & Wrist pronation/supination & -40 & 40 \\
\hline
\end{tabular}

belts, which supports wrist and hand. Given that the shoulder flexion/extension DOF is the most demanding in terms of torque, the exoskeleton is equipped with a passive antigravity system. Both the exoskeleton and the upper limb are sustained by a mounting frame, which is composed by various tubes and clamps. Such a structure can be fixed to different wheelchairs and it allows a correct positioning of the orthosis with respect to the user. The system is shown in Figure 2.

\section{B. Control Strategy}

The main control strategy takes advantage of position controllers installed at each joint motor. Once the initialization procedure has been completed and the exoskeleton has been donned, the user can drive the motion of the exoskeleton by directly controlling the position and orientation of the end-effector of the robotic system (i.e., the hand), that is reflected, thanks to an inverse kinematics algorithm, in position control strategies at each joint. More in detail, as shown in Figure 3, the target cartesian position of the hand $\left(\boldsymbol{x}_{\text {des }}\right)$ is computed at each iteration as the position at the previous step $\left(\boldsymbol{x}_{\text {curr }}\right)$ combined with a differential displacement obtained by the human-machine interfaces $\left(\boldsymbol{\Delta} \boldsymbol{x}_{\text {des }}\right)$. Then, an algorithm implementing the inverse model of the robot computes the desired angular position $\left(\boldsymbol{q}_{\text {des }}\right)$ that is set to the controller of the stepper motor. Moreover, during the free-use of the exoskeleton, the user has the possibility to save and retrieve peculiar positions by means of different human-machine interfaces. Further details have been described in previous works [9], [10].

\section{Human-Machine Control Interfaces}

The key concept concerning the control of the exoskeleton is to provide the user with a tool to drive the position of the end-effector in the workspace. This is achieved by means of different Human-Machine Control Interfaces that have been described and evaluated in a previous work [9]. Since the control of the exoskeleton has to be adapted to the level of disability, two interfaces were developed and tested in this study.

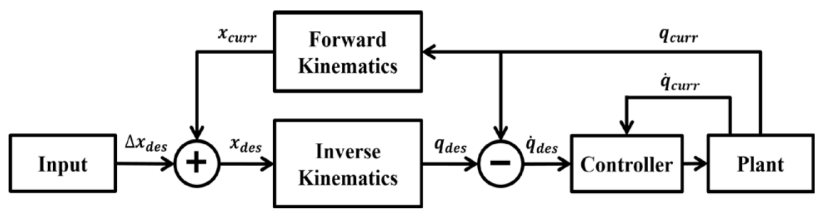

Figure 2. Cartesian Position Control Scheme 


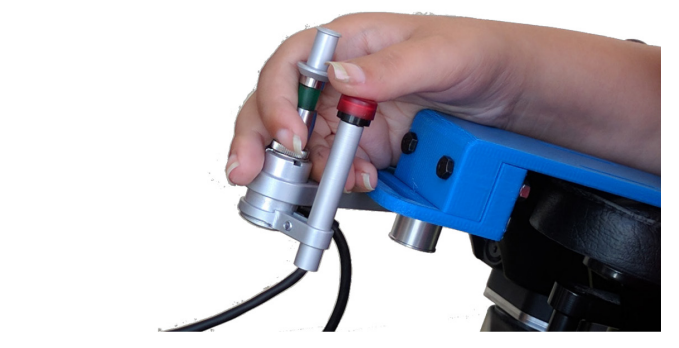

Figure 4. High sensitivity joystick (Ottobock, Ger)

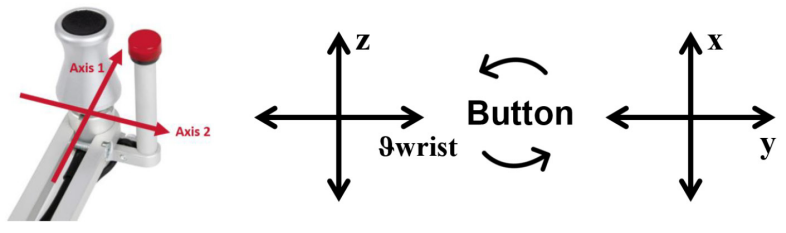

Figure 5. Joystick control scheme

1) The Manual Control Interface (MCI) is based on a 2-axis high sensitivity joystick (Ottobock, Ger) which is pictured in Figure 4. The joystick is manipulated with the contralateral hand with respect to the arm assisted by the device and it allows the user to control the $3 \mathrm{D}$ cartesian position of the hand. In particular, since the joystick only exploits two axis and the user has to move the exoskeleton in a 3D space, by clicking a dedicated push-button the patient is able to change from horizontal working plane to the vertical one (Figure 5).

2) The Vocal Control Interface (VoCI) continuously listens and detects hot-words and sentences recognizing vocal commands for the control of the exoskeleton. Six hot-words, corresponding to the six main directions in the threedimensional space, were used to control the exoskeleton.

\section{Participants}

Participants were recruited from in-patients and outpatients at IRCCS E. Medea and Rehabilitation Institute Villa Beretta. Eligible participants met these inclusion criteria: (1) availability to sign the informed consent, (2) defined diagnosis of Duchenne Muscular Dystrophy (DMD), Becker Muscular Dystrophy (BMD) or Limb Girdle type 2 Muscular Dystrophy (LGMD2), (3) wheelchair dependence, (4) Muscular Rating Council (MRC) score [11] at the deltoid and biceps brachii muscles ranging from 0 to 2, (5) no cognitive impairments and (6) arm length measured from the shoulder to the elbow between $26.5 \mathrm{~cm}$ and $28.7 \mathrm{~cm}$ and forearm length measured from the elbow to the wrist of maximum $22.4 \mathrm{~cm}$.

Exclusion criteria were: (1) presence of relevant comorbidities (for example: epilepsy, 24/24-hour dependence on non-invasive or invasive ventilation), (2) behavioral and psychiatric disturbances (for example: emotional problems, depression) and (3) absence of compliance to the study of the family and the caregivers.

\section{E. Outcome measures}

In order to assess upper limb function, the Performance of the Upper Limb scale (PUL) was selected as primary outcome measure. PUL has been recently designed for specifically assessing arm functionality in DMD patients
[12]. This scale is constituted of 22 items, subdivided into an entry level and three main levels reflecting disease progression from proximal to distal part of the arm: shoulder dimension (4 items), elbow dimension (9 items) and, wrist and finger dimension ( 8 items). The total score is obtained by adding the three scores, with a maximum of 74 points and a higher value corresponding to less impairment of the subject and vice versa.

Then, the self-perceived manual ability, defined as the capacity to use the hands and upper limbs to perform manual daily activities regardless of the strategies involved [13], was assessed with the ABILHAND questionnaire [14], specifically designed for neuromuscular patients. Participants were asked to provide their perceived difficulty in performing 22 activities. Possible answers to each item were: "easy", "difficult" or "impossible". This questionnaire was analyzed following Vandervelde and colleagues guidelines [14], who used the Rasch model to estimate the difficulty of each item and patients' self-perceived manual ability. Results are expressed in logit and they can range from -6.176 logit, if the subject considers all actions impossible to be performed, to +6.427 logit, if the patient believes to succeed in accomplish all items easily. Thus, higher scores represent higher patients perceived manual ability.

Finally, a quantitative evaluation of the usability of the exoskeleton was provided by the SUS questionnaire. It is a ten-items scale giving a global view of subjective assessments of usefulness, as a combination of effectiveness, efficacy and satisfaction of the system [15]. The global scores were computed according to Sauro's guidelines [16]. SUS scores have a range from 0 to 100 .

\section{F. Experimental protocol}

The clinical protocol describing the pilot study with IIa non-CE-marked device was approved by ethical committees of the involved centers, and by the Italian Ministry of Health on May $4^{\text {th }}, 2018$. The clinical protocol includes three days of testing (DAY 1, DAY 2 and DAY 3) in one of the two involved clinical centers. The following activities are foreseen for each day.

DAY 1 - A baseline evaluation of the upper limb motor functional scale is performed without the assistive device, including the following assessments: i) MRC scale, ii) PUL scale and iii) ABILHAND questionnaire. Afterwards, interaction modalities (MCI and $\mathrm{VoCI}$ ) to control the exoskeleton are presented to the patient and tested in a simulation environment. This procedure is aimed at identifying which human-machine interface the patient is able to use to control the device. Finally, technicians, together with a physiotherapist, mount and adjust the device to the patient's wheelchair and the exoskeleton is worn on the left arm of the patient. In this phase, the exoskeleton is customized to fit different users' arm dimensions.

DAY 2 - Participants are asked to perform a 2 hours session of task-oriented movements (e.g. reaching a mouse and objects on a table, moving back to the wheelchair, drinking, type on a computer keyboard, etc.), and free movements, accordingly to their residual capabilities. In this phase the participant also has to get used to his/her arm to move again, which usually requires a bit of time. 
DAY 3 - This phase has to be performed after DAY2, and in any case within 15 days. Each subject uses the exoskeleton for one hour. Afterwards, the final evaluation with the device has to be performed, including: PUL scale, ABILHAND and System Usability Scale (SUS).

\section{RESULTS}

\section{A. Participants}

Up to now, five eligible patients were recruited at IRCCS E. Medea (P1-4), and Rehabilitation Institute Villa Beretta (P5). Three patients had LGMD, and two had DMD. The mean age of participants was 31 years, ranging from 18 years to 53 years. Patients were severely impaired, with deltoid MRC values ranging from 0 to 1 , and biceps brachii MRC values between 0 and 1.5 .

\section{B. PUL scale results}

A physiotherapist performed the PUL scale at DAY 1 and at DAY 3 in order to evaluate the effect of BRIDGE/EMPATIA exoskeleton at the upper limb functional level (Figure 7). PUL scores obtained at DAY 1 were heterogeneous among patients, ranging from 2 to 42 points (Figure 6, Table 2). The median value at DAY 1 was equal to 17 points $(\mathrm{IQR}=$ 20.50). All patients increased their upper limb functional abilities with the exoskeleton (Table 2). The median of the global improvement with the exoskeleton was 12 points (IQR $=4.25$ ). The greatest enhancements were recorded at the elbow level, with a median of the difference in score between final and baseline evaluation equal to 8 points $(\mathrm{IQR}=4)$ over nine elbow-related items. At the wrist and hand level, a median improvement of 4 points $(\mathrm{IQR}=8.25)$ was obtained. Figure 6 represents PUL scores achieved by each patient without and with BRIDGE/EMPATIA exoskeleton, subdivided in elbow, wrist and finger levels. It has to be noted that shoulder-related points are not present in all patient. This is due to the fact that the entry item for the shoulder-related items has to be performed with two arms, which is impossible to be executed even if wearing the system.

\section{ABILHAND results}

ABILHAND test was administered to each subject at DAY 1 and at DAY 3, to evaluate the difficulty that patients perceived in carrying out activities of daily living without and with BRIDGE/EMPATIA exoskeleton. All patients increased their perceived manual ability with the exoskeleton, except for one patient (Table 2). The median of the difference between the baseline score and the final score was 0.86 logit $(\mathrm{IQR}=3.35)$, showing that patients self-perceived ability increased. In particular, without exoskeleton patients scored -1.85 logit, meaning that they considered seven items "difficult", while the remaining fifteen were considered "impossible". With the exoskeleton, instead, the median result was -0.98 logit, meaning only six items were evaluated as "impossible" to be performed, the other items, such as washing hands, unwrapping a chocolate bar, wiping someone hands, dealing cards or counting banknotes, were "difficult", but feasible.

\section{SUS scale results}

The evaluation of BRIDGE/EMPATIA exoskeleton usability was provided by the SUS questionnaire. Each patient filled
TABle 2. Pul, ABILHAND AND Sus SCORE OBTAINED By PATIENTS WITH AND WITHOUT THE EXOSKELETON

\begin{tabular}{|c|c|c|c|c|c|}
\hline \multirow{2}{*}{$\begin{array}{c}\text { Subject } \\
\text { ID }\end{array}$} & \multicolumn{2}{|c|}{$\begin{array}{c}\text { DAY1 } \\
\text { (Without exoskeleton) }\end{array}$} & \multicolumn{3}{|c|}{$\begin{array}{c}\text { DAY3 } \\
\text { (With exoskeleton) }\end{array}$} \\
\cline { 2 - 6 } & PUL & ABILHAND & PUL & ABILHAND & SUS \\
\hline 1 & 42 & 4.814 & 53 & 2.996 & 92.5 \\
\hline 2 & 17 & -1.845 & 29 & -0.983 & 75 \\
\hline 3 & 18 & -1.845 & 29 & -0.983 & 72.5 \\
\hline 4 & 4 & -6.176 & 18 & -1.398 & 87.5 \\
\hline 5 & 2 & -2.644 & 21 & -0.009 & 75 \\
\hline
\end{tabular}

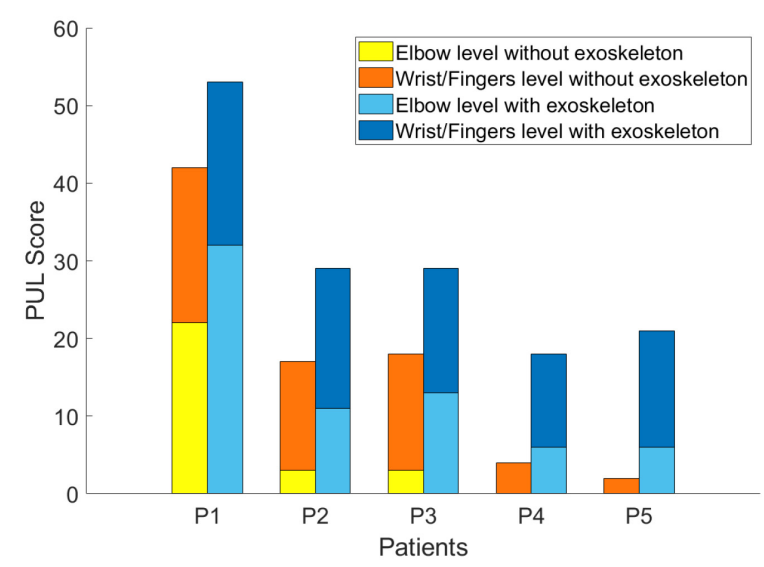

Figure 6. Total PUL score for each patient with and without the exoskeleton
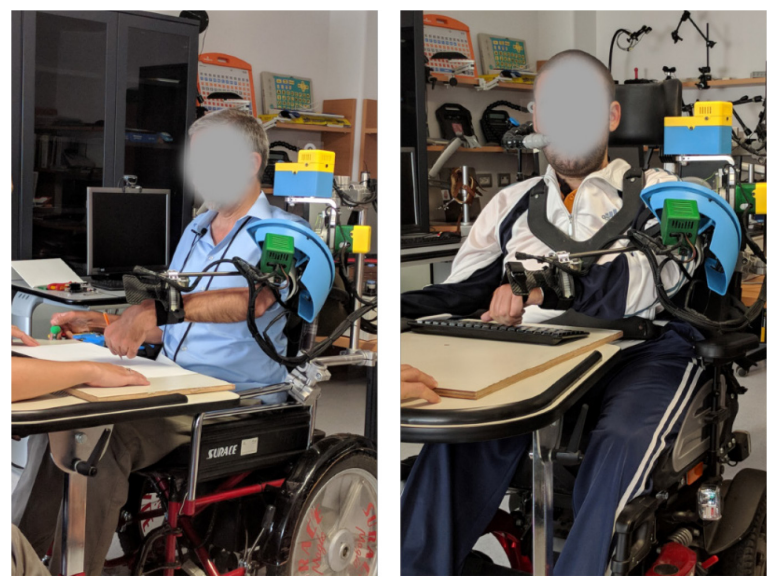

Figure 7. MD patients performing exercises during pilot study

the questionnaire at the end of DAY 3 (Table 2). SUS median score was $75(\mathrm{IQR}=14.38)$, showing an overall good usability of the exoskeleton [18].

\section{DISCUSSION}

Muscular Dystrophy is a severe neuromuscular disorder that leads to progressive and severely disabling muscle weakness and loss of functional abilities. People with these disorders experience limitations in performing daily life activities, in their independence and quality of life and they need to rely on assistance from caregivers. The BRIDGE/EMPATIA system was developed with the aim of helping people with Muscular Dystrophy in the assistance and support of arm functions, allowing movements 
throughout the 3-dimensional workspace under the direct control of the user him/herself. The objective of this study was to assess the effect and the perceived functional benefit of this arm support, together with its usability and ease of use. Up to now, the device has been tested on five end-users with Muscular Dystrophy. The obtained results were encouraging, and most of the subjects found the exoskeleton to be beneficial. According to the objective functional benefit evaluation performed through the PUL scale, all participants increased their range of motion and were able to perform activities that were not possible without the exoskeleton, in line with the ones found by Kooren and colleagues [17], who evaluated the effect of a passive arm support, that compensates for the arm weight using elastic bands, on three DMD subjects through the PUL scale. Examples of functional tasks possible with the BRIDGE/EMPATIA exoskeleton include ability to reach the face, to drink, to eat or to comb hair. These results show that subjects' ability to perform everyday tasks considerably improved while wearing the exoskeleton. This is especially perceived by patients who have more severe limitations in upper-extremity performance. In fact, the subjects who found the exoskeleton most beneficial were patient P4 and P5, who were by far the most impaired (as detected by DAY 1 PUL scores). In contrast, the patient with the highest PUL starting score decreased his ABILHAND score, suggesting that subjects characterized by less severe disability tend to stick with their compensatory movements techniques to overcome limitations rather than exploit an additional device. When coming to usability of the device, an overall good usability was recorded. SUS scores were higher than 68 for each patient, so according to Sauro [16] these results are above average. A median value of 75 was obtained, and according to Bangor et al. [18] it can be classified as "good". Patients considered BRIDGE/EMPATIA useful and easy to be used. They also thought that all its functionalities were well integrated. Concerning the control interfaces, every patient was able to use all the possible interfaces. Four patients preferred the Manual Control Interface, which is very similar to the joystick used to move the electric wheelchair. One patient preferred the VoCI since he was used to interface with a similar vocal control with his mobile phone.

The BRIDGE/EMPATIA pilot study aims at recruiting fourteen end-users affected by muscular dystrophy to picture a more clear hypothesis of the effects of the designed exoskeleton on upper limbs functionality. Anyhow, further work is needed toward the characterization of the impact of a fully actuated upper limb exoskeleton on the activity of daily life of severely impaired people.

\section{CONCLUSION}

The experimental protocol investigating the use of the BRIDGE/EMPATIA system was performed with five real endusers and it led to encouraging results. Upper limb functional ability increased, as detected by objective functional measurement, and patients reported self-perceived usefulness. System usability has been evaluated to be good.

\section{ACKNOWLEDGMENT}

This work is funded by BRIDGE and EMPATIA projects, funded by Fondazione Cariplo in collaboration with
Regione Lombardia. The research is constantly supported by the UILDM association (Unione Italiana Lotta contro la Distrofia Muscolare, and by patients that agreed to participate for free to the project.

\section{REFERENCES}

[1] A. Antonietti et al., "Clinical Benefits and Acceptability of Two Commercial Arm Exoskeletons for Patients with Muscular Dystrophy," Springer, Cham, 2019, pp. 31-35.

[2] L. A. Van Der Heide, B. Van Ninhuijs, A. Bergsma, G. J. Gelderblom, D. J. Van Der Pijl, and L. P. De Witte, “An overview and categorization of dynamic arm supports for people with decreased arm function," Prosthetics and Orthotics International, vol. 38, no. 4. pp. 287-302, 15-Aug-2014.

[3] A. Bergsma et al., "1st Workshop on Upper-Extremity Assistive Technology for People with Duchenne: State of the art, emerging avenues, and challenges. April 27th 2015, London, United Kingdom.," in Neuromuscular Disorders, 2016, vol. 26, no. 6, pp. 386-393.

[4] A. Bergsma, M. M. H. P. Janssen, A. C. H. Geurts, E. H. C. Cup, and I. J. M. de Groot, "Different profiles of upper limb function in four types of neuromuscular disorders," Neuromuscul. Disord., vol. 27, no. 12, pp. 1115-1122, Dec. 2017.

[5] M. J. Jan Burgers, J. Burgers, M. Jannink, N. van Alfen, and I. J. de Groot, "Upper Limb Training with Dynamic Arm Support in Boys with Duchenne Muscular Dystrophy: A Feasibility Study," Int. J. Phys. Med. Rehabil., vol. 03, no. 02, p., Feb. 2015.

[6] A. Pedrocchi et al., "MUNDUS project: MUltimodal Neuroprosthesis for daily Upper limb Support," J. Neuroeng. Rehabil., vol. 10, no. 1, p. 66, Jul. 2013.

[7] E. Ambrosini et al., "A myocontrolled neuroprosthesis integrated with a passive exoskeleton to support upper limb activities," $J$. Electromyogr. Kinesiol., vol. 24, no. 2, pp. 307-317, Apr. 2014.

[8] M. P. W. Reichenfelser, "Arm exoskeleton, Patent WO 2016/187636 A1," Patent WO 2016/187636 A1, 2016.

[9] S. Dalla Gasperina et al., "Multi-Modal Human-Machine Control Interfaces of Upper Limb Motorized Exoskeletons for Severely Impaired Patients," in Proceedings of the IEEE RAS and EMBS International Conference on Biomedical Robotics and Biomechatronics, 2018, vol. 2018-Augus, pp. 491-496.

[10] M. Gandolla et al., "BRIDGE - Behavioural reaching interfaces during daily antigravity activities through upper limb exoskeleton: Preliminary results," in IEEE International Conference on Rehabilitation Robotics, 2017, pp. 1007-1012.

[11] A. Compston, "Aids to the investigation of peripheral nerve injuries. Medical Research Council: Nerve Injuries Research Committee. His Majesty's Stationery Office: 1942; pp. 48 (iii) and 74 figures and 7 diagrams; with aids to the examination of the peripheral nervous ," Brain, vol. 133, no. 10, pp. 2838-2844, Oct. 2010.

[12] A. Mayhew et al., "Development of the Performance of the Upper Limb module for Duchenne muscular dystrophy," Dev. Med. Child Neurol., vol. 55, no. 11, pp. 1038-1045, Nov. 2013.

[13] M. Penta, J. L. Thonnard, and L. Tesio, "ABILHAND: a Raschbuilt measure of manual ability.," Arch. Phys. Med. Rehabil., vol. 79, no. 9, pp. 1038-42, Sep. 1998.

[14] L. Vandervelde, P. Y. K. Van Den Bergh, M. Penta, and J. L. Thonnard, "Validation of the ABILHAND questionnaire to measure manual ability in children and adults with neuromuscular disorders," J. Neurol. Neurosurg. Psychiatry, vol. 81, no. 5, pp. 506-512, May 2010.

[15] J. Brooke, "SUS: A Retrospective," J. Usability Stud., vol. 8, no. 2, pp. 29-40, 2013.

[16] J. Sauro, A Practical Guide to the System Usability Scale ( SUS ). 2011.

[17] P. N. Kooren et al., "Design and pilot validation of A-gear: a novel wearable dynamic arm support," J. Neuroeng. Rehabil., vol. 12, no. 1, p. 83, Dec. 2015.

[18] A. Bangor, P. T. Kortum, and J. T. Miller, "An empirical evaluation of the system usability scale," Int. J. Hum. Comput. Interact., vol. 24, no. 6, pp. 574-594, Jul. 2008. 